\title{
Peripheral Arterial Occlusive Disease
}

National Cancer Institute

\section{Source}

National Cancer Institute. Peripheral Arterial Occlusive Disease. NCI Thesaurus. Code C78533.

Disorder caused by the occlusion of the lumen of the peripheral arteries. Causes include atherosclerosis, inflammatory processes, thrombosis, and embolism. The arterial occlusion results in chronic or acute pain usually in the lower limbs due to muscle ischemia. 